\section{Facts, not rhetoric on yellow rain}

SIR - R.D. Caldwell, in his letter “' 'Yellow rain' or natural toxins?'”(ref.1), comments and expands on the feasibility of naturally occurring mycotoxins in SouthEast Asia and advocates the open exploration of alternative hypotheses instead of the trading of unfounded accusations.

It is unfortunate that Caldwell, in his eagerness to get his point across, makes use of unfounded arguments himself. He refers in his letter to Canadian investigations ${ }^{2}$ in which, according to Caldwell, it is reported that trichothecene-producing fungi, such as Fusarium semitectum and Fusarium sporotrichioides, were found in most samples collected near the ThailandKampuchea border.

While the report ${ }^{2}$ is not very specific as to the actual number and types of isolates (this work is still in progress and not yet published), nowhere in the report does it say that $\boldsymbol{F}$. sporotrichioides was isolated. I presume that Caldwell misinterpreted the tentative identification (page 25 of the report) as the final diagnosis. In fact, the text on page 25 continues by stating explicitly that this suspected strain of $F$. sporotrichioides was finally classified as $F$. semitectum.

Also, I regret Caldwell's premature conclusion that the isolates were trichothecene-producing fungi. It is well known that the identification of a particular fungus does not give any clues as to the toxigenic potential. The toxigenic potential of the isolates of the Canadian study has not been determined yet, thus we do not know what potential they might have. However, one should recognize at this point the fact that the chemical analysis of the environmental samples from which the fungi were isolated did not reveal the presence of any mycotoxins. (Incidentally, $F$. semitectum was isolated only once from all these samples.) The only possible hint that toxigenic Fusarium spp. may exist in Thailand can be found in a recent publication ${ }^{3}$, in which it is stated that an extract of a culture of $F$. semitectum isolated from a "yellow rain" spot caused death of experimental animals, but the chemical analysis of the extract remains to be done. Another article in Nature ${ }^{4}$ misquotes this Thai paper by stating that " $F$. tricinctum, which produces the mycotoxin T-2"' was found in 22 of the specimens of yellow rain. What the article actually says is that $F$. semitectum (not $F$. tricinctum) was not found in 25 sampling places on the leaves apart from the yellow rain spots.

Further, I am rather critical of Caldwell's statement that T-2 producing Fusarium spp. were described in the 1930s in Vietnam. At that time, nobody knew of T-2 toxin, and all that one can say is that Fusarium species, which might be able to produce $\mathrm{T}-2$ toxin, were found in Vietnam in the $1930 \mathrm{~s}$, but their toxigenicity was never assessed.

In view of the established facts, I find it premature to conclude, as Caldwell does, that trichothecenes occur naturally in South-East Asia. I find it even more premature to conclude that the events that are described as occurring in Kampuchea and Laos can be explained on the basis of natural occurrence of toxins.

I invite Caldwell and anyone who has the scientific capability and resources to conduct the much needed studies, that is, to investigate the matter of natural occurrence of mycotoxins, particularly trichothecenes, in South-East Asia. It is not rhetoric or scepticism, which leads to misinterpretation of data, that will help solve this problem; what we need are hard scientific facts.

\section{Toxicology Research Centre,}

\section{University of Saskatchewan,}

Saskatoon, Saskatchewan, Canada

1. Caldwell, R.D. Nature 301, 651 (1983).

2. Schicfer, H.B. Canadian Submission to the UN, A37/308 (1982)

3. Sukroongreung, S, et al. Siriraj Hosp. Gaz. 34, 643-647 (1982).

4. Budiansky, S. Nature 302, 200-201 (1983).

\section{Vote for A?}

SIR - R.M. May' raises again the difficulties facing a system of voting to fill a single place, and in particular the paradox (A beats $B, B$ beats $C, C$ beats $A$ ) that can arise if Condorcet's system of pairwise comparisons is used. He writes, however, that his general impression is that such common in practice".

I should like to comment, as someone who has used Condorcet voting in practice, mainly within the council of the Royal Statistical Society. In more than a dozen uses I have never met a circularity for the first place yet. Of course, such a result, or a tie, could occur and rules must be drawn up for what happens next if so, but this is no worse than the fact that a tie could occur in any voting system.

Where election is to fill a single position, such as a president, a pope or a party leader, it has enormous advantages over any other system, namely: (1) It completely eliminates the effect of "split" votes and tactical voting (unless the paradox intrudes). (2) It is therefore very easy for the voter to use, as the only sensible ordering of the candidates is by true preference. (3) Anyone can stand for election without worrying about spoiling someone else's chances relative to any other candidate. (4) Unless the paradox arises it gets the right result (that is, the candidate who could have won a straight fight against any one of the others) and does so in only one round of voting. (5) If the paradox arises it still does not give the wrong result but is merely indeterminate. paradoxical results "are not likely to be too
The difficulty lies not in the system itself but in convincing people that it is the right system to adopt. The situation is not helped by the publication of a recent book by R.A. Newland ${ }^{2}$ which includes a chapter on "Filling a single place". This chapter correctly shows that no system is really worth consideration except Condorcet or the "alternative vote" method, but then says "the alternative vote is the preferred method", without mentioning any of Condorcet's advantages. As the author is a past chairman of the Electoral Reform Society, his advice may unfortunately be taken seriously.

It should be noted that Condorcet is not a contender for electoral reform where proportionality is required. It is no more a proportional system than is our present parliamentary electoral system. It can be used, however, to elect more than one candidate where proportionality is not required. For example, it would be ideal for electing, or re-electing, four teams each year to the Fourth Division of the Football League.

It is sometimes contended that the counting is difficult. In practice, it is much easier than might be thought at first glance, but in any case that should not really be a consideration nowadays when a computing algorithm ${ }^{3}$ can be written to do the task.

Clinical Research Centre,

I.D. HILI.

\section{Harrow, UK}

1. May, R.M. Nature 303, 16-17 (1983).

. Newland, R.A. Comparative Electoral Systems (The Arthur McDougall Fund, London, 1982).

3. Hill, I.D. Computer J. 18, 89-90 (1975).

\section{Foothills and corridors}

SIR - Concerning Sydney Brenner's pessimistic vision of molecular biology (Nature 21 April, p.654), may I offer a countervision - that on cloudy or hazy days we can but see "the foothills" despite the nearby ranges; and that the successive "long corridors" which are molecular biology today, and which have doors only at the distal ends, may also have high windows, or cracks in the walls, such that only those tall enough or sufficiently astute can see out. Ultimate liberation might be the privilege of those who attempt to break away from the constraints of the corridor by enlarging the cracks or by going through the window (vandalism, of course). Isn't this exactly a "paradigm-shift", the concept you assail (Nature 14 April, p.557)? In this context what was ascribed to Brenner should perhaps read: "[the long-corridors story] is what normal science is like". Understandably, what one can do in Kuhnian normal science is "just [to] publish more and more. . .".

A. Sibatani

CSIRO Molecular and Cellular

Biology Unit,

Sydney, NSW, Australia 